\title{
No evidence for stopping long-term aspirin therapy before tooth extraction
}

\author{
Abstracted from \\ Zhao B, Wang P, Dong Y, Zhu Y, Zhao H. \\ Should aspirin be stopped before tooth extraction? A meta-analysis. \\ Oral Surg Oral Med Oral Pathol Oral Radiol 2015; 119: 522-530. \\ Address for correspondence: H. Zhao College of Stomatology, Shandong University, \\ Number 44, Wen Hua Xi Lu, Jinan City, Shandong Province 250012, China. \\ E-mail: Zhaohq@sdu.edu.cn
}

\section{Question: Should long-term aspirin should be stopped before tooth extraction?}

Data sources PubMed, ScienceDirect and EBSCOhost databases. Study selection Prospective randomised controlled trials (RCTs) or controlled trials.

Data extraction and synthesis Two independent investigators extracted data. The primary measured outcomes were rates of haemorrhage and bleeding time. Disagreements were clarified with a third investigator. Relevant authors were contacted if any relevant data was missing. The Grades of Recommendation, Assessment, Development and Evaluation (GRADE) system was used to evaluate the overall quality of evidence. The authors used standardised mean difference or relative risk to evaluate each outcome.

Results Ten studies were included, three randomised controlled trials and seven controlled trials. A total of 1752 patients were enrolled in the meta-analysis; the study group comprised 529 patients on longterm aspirin therapy, and the control group comprised 1223 patients. The risk of post-operative haemorrhage was significantly higher in patients on aspirin therapy (relative risk $=2.46 ; 95 \%$ confidence interval: 1.45-4.81) but bleeding time was not significantly different between the two groups (standardised mean difference $=0.63 ; 95 \%$ Cl: - 0.04 to 1.31 ).

Conclusions Increased rates of haemorrhage are observed in patients on long-term aspirin therapy. The authors recommend not stopping long-term aspirin prior to dental extraction, and local measures for haemostasis should be enhanced when required.

\section{Commentary}

Aspirin is a drug commonly prescribed for its analgesic, antipyretic, and anti-inflammatory properties. It is also often prescribed for its anti-platelet properties for the prevention of cardiovascular disease. ${ }^{1}$ This inhibition of platelet aggregation results in prolonged bleeding times and delayed primary haemostasis. ${ }^{1}$ The Scottish Dental Clinical Effectiveness Programme, (SDCEP), in its guidance document does not advise stopping aspirin for dental extraction. ${ }^{1}$ Stopping aspirin can increase the patient's risk of a thromboembolic event, and therefore local measures for haemostasis are preferable. ${ }^{1}$ The SDCEP guidance advises that dentists should be familiar with local haemostatic measures such as placement of haemostatic packing material and suturing and in cases such as multiple extractions or where complications are anticipated treatment should be performed over multiple visits. ${ }^{1}$ The risk of a thromboembolic event must be weighed carefully against the risk of haemorrhage.

The authors of this meta-analysis attempted to evaluate the evidence on whether aspirin should be stopped before tooth extraction. Although the rationale for the study was clear, the objectives of the study did not follow the Population, Intervention, Control and Outcome (PICO) format. There was no mention of the authors following a protocol in the systematic review and a search of The University of York Centre for Reviews and Dissemination database (PROSPERO) revealed no registration of this review. ${ }^{2}$ This may have affected the reproducibility of this study. The authors searched only Chinese and English papers, and did not seem to search any relevant journals by hand. This is a pity as the narrowing of the search may have introduced language bias into a useful review with a clinically relevant question.

The authors concede that there are a number of limitations to their meta-analysis including variation in methodologic quality, and heterogeneity due to different clinical interventions between studies, and different techniques measuring bleeding times. Some of the studies included surgical extractions, others only simple extractions, with variations in the number of teeth extracted. In addition, within the papers there is a wide range of aspirin dosage (75-325mg) with two of the studies not having a stated aspirin dose. The study by Lillis et al. had one of the highest patient numbers $(\mathrm{n}=574)$ of all the studies, and is non-randomised. ${ }^{3}$

Heterogeneity between the studies was assessed using the $\mathrm{I}^{2}$ statistic. Ideally for meta-analysis the heterogeneity (differences) between studies should be as minimal as possible so that their results can be combined. In this review it can be argued that there was 
significant heterogeneity to the point where it must be questioned whether a meta-analysis of the included studies was appropriate and likely to yield a valid result.

The overall quality of evidence was assessed as moderate risk of bias using Cochrane's GRADE system. The authors did not however assess the risk of bias within the studies. This is concerning considering seven of the studies were non-randomised and three were randomised. The patient numbers in the randomised trials (1614 patients) were considerably less than the non-randomised numbers with 138 patients. Randomisation in clinical studies is important to reduce bias, particularly allocation and selection bias. In this respect it is possible that some of these non-randomised studies may have been at high risk of bias.

The overall synthesis of the results was good. Standardised mean difference or relative risk with $95 \%$ confidence interval was used to evaluate each outcome. The relative risk was calculated using the random effect model in studies exhibiting heterogeneity; otherwise the fixed effect model was used. The standard mean difference was calculated using the random or fixed effect models.

Sensitivity analysis is useful to understand the impact an actual outcome of a particular variable will have if it differs from what was previously assumed. ${ }^{4}$ In this study for example it would have been useful to understand what would happen to bleeding time if metaanalysis were performed with just a single extraction compared with a multiple extraction, or for example how the bleeding time would differ if aspirin were used in different age groups etc. Unfortunately although sensitivity analysis was performed, it was not used for these variables. As part of the sensitivity analysis the authors included and excluded various studies from their analyses at different stages. The rationale for excluding the Dudek et al. (2013) and Lu et al. (2014) studies is explained; however the authors fail to explain the rationale for excluding the Ardekian et al. (2000) and Krishnan et al. (2008) studies. 5,6,7,8 This makes interpretation of the results somewhat confusing.

\section{Practice points}

- The authors conclude that long-term aspirin should not be stopped prior to dental extraction but practitioners should ensure they consider other methods of haemostatic control. This systematic review is in line with current guidance. ${ }^{1}$

Daphne Halley, Robert Weld-Moore and Brett Duane Dublin Dental University Hospital, Dublin, Ireland

1. Scottish Dental Clinical Effectiveness Programme. Management of Dental Patients Taking Anticoagulants or Antiplatelet Drugs, Dental Clinical Guidance. 2015. Online information available at http://www.sdcep.org.uk/published-guidance/ anticoagulants-and-antiplatelets/ [Accessed $10^{\text {th }}$ November 2015].

2. The University of York Centre for Reviews and Dissemination database. Online information available at http://www.york.ac.uk/crd/ [Accessed $10^{\text {th }}$ November 2015]

3. Lillis T, Ziakas A, Koskinas K, Tsirlis A, Giannoglou G. Safety of dental extractions during uninterrupted single or dual antiplatelet treatment. Am J Cardiol 2011; 108: 964-967.

4. Sensitivity Analysis Definition | Investopedia. Online information available at http:// www.investopedia.com/terms/s/sensitivityanalysis.asp\#ixzz3sV6VorAc [Accessed $10^{\text {th }}$ November 2015].

5. Dudek D, Helewski K, Wyrobiec G, et al. Therapy with acetylsalicylic acid does not interfere with oral surgery. Central Eur J Med 2013; 8: 392-397.

6. Lu MM, Zhuang XH, Gao YM. Correlation between platelet aggregation rates of aspirin users and intra-socket clotting after extraction of a maxillary tooth with periodontitis. Shanghai J Stomatol 2014; 23: 328-332.

7. Ardekian L, Gaspar R, Peled M, Brener B, Laufer D. Does low-dose aspirin therapy complicate oral surgical procedures? / Am Dent Assoc 2000; 131: 331-335.

8. Krishnan B, Shenoy NA, Alexander M. Exodontia and antiplatelet therapy. J Oral Maxillofac Surg 2008; 66: 2063-2066.

Evidence-Based Dentistry (2015) 16, 118-119. doi:10.1038/sj.ebd.6401137 